\title{
Detection of Zoonotic Rotavirus Transmission involving a Rare Genotype G3P[3] in the Amazon Region: A Phylodynamic Approach with Multiple Events of Reassortment and Evolutionary Origin
}

\author{
Renato da Silva Bandeira ${ }^{1}$, Lizandra Caroline dos Santos Souto ${ }^{1}$, Layse Costa de Souza ${ }^{1}$, \\ Delana Andreza Melo Bezerra ${ }^{2}$, Poliana da Silva Lemos ${ }^{2}$, Alexandre C. Linhares ${ }^{2}$ and \\ Joana D'Arc Pereira Mascarenhas ${ }^{2 *}$ \\ ${ }^{1}$ Department of Virology, Evandro Chagas Institute, Ananindeua-Pará-Brazil \\ ${ }^{2}$ Evandro Chagas Institute, Health Surveillance Secretariat, Brazilian Ministry of Health, \\ Ananindeua-Pará-Brazil \\ *Corresponding author
}

A B S T R A C T

\section{Keywords}

Rotavirus A,

Zoonotic, Rare,

Phylodynamic,

Amazon

Article Info

Accepted:

22 October 2019

Available Online:

10 November 2019
Rotaviruses of the species A (RVA) are of particular epidemiological importance and recognized as being worldwide dispersed. RVA causes the majority of infections in humans, as well as are highly prevalent among domestic animals. Several studies have shown that zoonotic transmission may occur from animals to humans and this may account for the current broad antigenic and genetic rotavirus diversity, representing a potential important evolutionary mechanism. The present study aims to identify the evolutionary origins of circulating RVA strains in the Amazon region, using phylodynamics methods to assess and understand the relationship between hosts, inter-specific gene transfer and the chronology of viral infection. In total 83 RVA-positive faecal samples of human origin were analyzed, all of which obtained within the official Rotavirus Epidemiological Surveillance Network in Brazil. The genome of 17 RVA samples were selected and sequenced. Eight RVA strains of zoonotic origins were identified. Of the five G4P[6] genotype samples were shown to be of porcine origin, and two samples were generated from reassortment events involving genotypes of human and porcine strains. One sample of G3P[3] genotype strain, identify as lineage III, have evolved from canine, feline or simian origin, including a reassortment event with RVA strains from lineage II. In addition, G12P[9] strains had their origins in chiroptera, cattle or felines. The present study included phylodynamic analyses in order to elucidate those otherwise unknown evolutionary patterns, mainly in regards to G3P[3] and G12P[9] genotypes. There was a particular focus on the occurrence of reassortment events and the evolutionary mechanism which underlie the emergence of these strains. These evolutionary events may help in the monitoring of emergent strains of RVA with zoonotic potential and vaccine-escape possibility.

\section{Introduction}

Rotaviruses belong to the Reoviridae family based on both shared genomic and morphological characteristics. To date nine groups or species of rotavirus have been described and classified as A to I, recently the newly specie $\mathrm{J}$ has been proposed (Bányai et 
al., 2017; Mihalov-Kovács et al., 2015). RVA, $\mathrm{RVB}, \mathrm{RVC}$ and RVH species are known to infect humans and animals, whereas other species infect animals only (Gomes et al., 2014; Kindler et al., 2013; Matthijnssens and Van Ranst, 2012; Trojnar et al., 2013). Rotaviruses of the species A (RVA) are of particular epidemiological importance and known to be of worldwide occurrence. In fact, RVA causes the majority of infections in humans as well as in domestic animals (Dóró et al., 2015; Tate et al., 2016).

The RVA genome is composed of 11-strand double-stranded RNA segments which encode 12 structural proteins (VP1-VP4, VP6 and VP7) and six non-structural proteins (NSP1 to NSP5/6) making up a triple-layered shell (Estes et al., 2013; Marton et al., 2017). Until recently the classification was based on a binary combination that gathered VP7 (G) and VP4 (P) genotype specificities. The newly adopted classification of rotaviruses relies on the characterization of the 11 RNA segments, as proposed by Matthijnssens et al., (2008). This full genome assessment provides a specificity for each gene segment, and allows for a better understanding of the evolutionary dynamics within this group of rotaviruses.

According to the new classification system a full genetic characterization of the RVA strains denoted different constellations gathering specificities for all eleven RNA segments. In this classification the acronym Gx-P[x]-Ix-Rx-Cx-Mx-Ax-Nx-Tx-Ex-Hx is utilized to define genotypes of the VP7-VP4VP6-VP1-VP2-VP3-NSP1-NSP2-NSP3-

NSP4-NSP5 genes, respectively (Matthijnssens et al., 2011). Three distinct RVA constellations have been defined to date including the WA-like, DS-1-like and AU-1like constellations which correspond to lineages I, II and III, respectively. Currently the number of genotypes identified for each gene is as follows: $\mathrm{G}(\mathrm{n}=36), \mathrm{P}(51), \mathrm{I}(26), \mathrm{R}$
(22), C (20), M (20), A (31), N (22), T (22), E (27) and $\mathrm{H}$ (22). As a result of utilizing this new classification system a growing number of sequences have continuously been submitted to the GenBank, allowing the description of numerous RVA genotype constellations of RVA for humans; in addition, this system has improved the identification of host species for several uncommon human RVA strains (Matthijnssens and Van Ranst, 2012).

In light of the whole viral genome characterization, several studies have been conducted worldwide (Gentsch et al., 2005; Khamrin et al., 2006; Martela et al., 2010), including Brazil (da Silva et al., 2011; Santos et al., 1999) and the Amazon region (Bezerra et al., 2017; Guerra et al., 2016; Maestri et al., 2012; Mascarenhas et al., 2007) which have reported the occurrence of rotavirus strains of human/animal origin, suggesting interspecies transmission. In general, it has been considered rotavirus of animal origin as a possible reservoir contributing for human rotavirus antigenic and genetic diversity, the potential for interspecies transmission from animals to humans or vice versa is believed to account for genetic diversity and evolution (Dóró et al., 2015).

The great diversity of circulating rotavirus and the characteristic of its segmented genome, allows easy reassortment of cells co-infected with strains of different origins (Midgley et al., 2014). Studies in the Amazon region concerning the origin and evolution of rotavirus strains with zoonotic potential are still limited.

In this region the poor sanitary conditions and the presence of domestic and wild animals in close proximity to humans are factors that may account for the emergence of strains resulting from frequent interspecies reassortment events involving rotaviruses of 
human and animal origins (Dhama et al., 2009; Maestri et al., 2012; Nakagomi et al., 2018; Wolfe et al., 2007).

The continuous monitoring of circulating RVA strains is of particular epidemiological importance in the current post-rotavirus vaccine introduction era. Indeed, the potential for emergence of either new or unusual rotavirus may represent a challenge to current vaccination strategies (Matthijnssens et al., 2008). The present study aims to identify the evolutionary origins of circulating RVA samples in the Amazon region using phylodynamic methods to allow for a better understanding of the relationship between hosts, inter-specific gene transfers and the chronology of evolution dynamics.

\section{Materials and Methods}

\section{Ethical considerations and study design}

The present study was approved by the Ethics Committee of Research in human beings of the Evandro Chagas Institute, Health Surveillance Secretariat, under the registration number 2.587.984/2018. Overall 83 RVApositive fecal samples were obtained from three (A, B and C) studies conducted previously in Belém, Northern Brazil.

Study A involved infants and young children hospitalised for acute gastroenteritis in a local paediatric hospital between 1990 and 1992 (Linhares et al., 1996; Mascarenhas et al., 2002); Study B included children who were either hospitalized for acute diarrhoea or sought for medical treatment at an outpatient health unit in the context of a two-year surveillance study in Belém, between May 1998 and May 2000 (Gabbay et al., 2007); and Study C which was essentially an epidemiological pre-rotavirus vaccine trial carried out throughout 2003 (Soares et al., 2010).
Selection of samples, extraction, PCR and sequence

The inclusion criterion for this study was similar to that followed by Maestri et al., (2012), who had selected samples which were previously genotyped for VP7, VP4, VP6 and NSP4 genes yielding genotypes that might indicate potential zoonotic transmission including G3P[3], G3P[6], G3P[9] and G12P[9]. An alternative criterion used in this study was based on a previous phylogenetic analyzes performed by Maximum Likelihood that would allow selection of potential zoonotic strains identified in a previous in silico analysis.

Stool samples were prepared as $10 \% \mathrm{v} / \mathrm{v}$ suspension in $0.01 \mathrm{M}$ Tris- $\mathrm{Ca}^{++}$buffer $\mathrm{pH} 7.2$, followed by extraction of viral RNA in silica (Boom et al., 1990). After extraction RT-PCR was performed in two steps. The first step consisted of obtaining Replication Transcription of the DNA strand (cDNA) and the second step aimed to obtain amplicons by PCR, using specific primers for VP7, VP4, VP6 and NSP4 genes. The amplicons were further analyzed through $1.5 \%$ agarose gel electrophoresis. The resulting PCR products were then purified followed by sequencing using the ABI Prism 3130 automated sequencer (Applied Biosystems; Foster City, CA). Samples that yielded a viral log greater than $50 \mathrm{ng} / \mu \mathrm{L}$ were sequenced using the Ion torrent $\mathrm{PGM}^{\mathrm{tm}}$ platform (Life Technologies; Carlsbad, US).

\section{Phylodynamics analysis}

Sequences obtained from studied genes were subsequently assembled with reference sequences deposited at the GenBank for reliability, followed by their editing utilizing the Geneious v.10.0.6 program (Kearse et al., 2012), aligned with the Mafft v.7 program (Katoh, 2013), and implemented within the 
Aliview v.1.17.1 program (Larsson, 2014). The elimination of recombinant strains was performed by the RDP4 programs v.4.46 (Martin et al., 2015) using 12 parameters, by SimPlot v.3.5.1 (Lole et al., 1999) and by SplitsTree v.4.14.4 (Huson and Bryant, 2005) by the phyTest method. In this program we have also evaluated the proportion of invariant sites.

For the previous phylogenetic reconstruction, the maximum likelihood method was used in the IQ-Tree v.1.0.1 program with 1000 replicas of bootstrap (Nguyen et al., 2014). These results were evaluated using the TempEst v.1.5 program (Rambaut et al., 2016) to observe the correlation of samples with the molecular clock test by the $\mathrm{R}^{2}$ value. Phylodynamics was performed in the BEAST v.1.8 program (Suchard et al., 2018) by Bayesian inference. Initially a simple run was performed to test the three clock models and four coalescing models. In order to determine the best model (clock/coalescence), the "Model Comparison" method was used in the Tracer v.1.6 program (Rambaut et al., 2014) in which the lowest A.I.C.M. value determines the best evolutionary model for the analyzed bank. Subsequently, a more robust race with 4 chains was performed using the best indicated model added from the geographic and host data of origin.

The tests of the best models and the results generated by BEAST were analyzed again in Tracer. The graphic result was visualized using the program Fig Tree v. 1.4.2 (Rambaut, 2009), which generates the graphical visualization of the phylogeographic tree with the clock data, modeling the geographical distribution and the most probable origin host.

The nucleotide sequences were deposited in the GenBank under the accession numbers MK272854-MK272935, MN082045, DQ525195 and DQ525185.

\section{Results and Discussion}

\section{Zoonotic potential}

Were selected 17 of the 83 study samples to genomic analysis. Phylogeny and determination of nucleotide similarities indicated that $53 \%(9 / 17)$ of the samples had a potential zoonotic origin, whereas $47 \%(8 / 17)$ represented human genotypes belonging to the WA-like and DS-1-like genogroups. Among the potential zoonotic samples six were partially characterized whereas full genomic characterization could be done in three samples (52127, 56010 and 55018) (Table 1).

Comparative analyzes indicated that potential zoonotic samples were grouped in the within WA-like, DS-1-like and AU-1-like constellation (I, II and III lineages) bearing G3P[3], G4P[6] and G12P[9] genotypespecificities. Samples with G4P[6] genotypes related to samples belonging to lineages I and II, suggesting an evolution event mainly from porcine origin. The sample COD424 showing a reassortment in the NSP3 gene genotype T7 and the sample HSE005 present reassortment in the NSP4 gene with strain with genotype E1 origin, lineages I. The G3P[3] sample displayed possible canine and feline origins, through reassortment events including the NSP2 and NSP5 genes (N2 and H6, respectively). Samples with G12P[9] genotype-specificity, were recovered from Chiroptera, or of either bovine or feline origin. Of these, two strains showed reassortment involving the NSP5 gene (H6 genotype). No recombinant sample was detected (Table 1).

\section{Phylodynamics}

With regards to the G3P[3] and G12P[9] samples which displayed an unusual genotype combination pattern and an unusual evolutionary origin, a further more accurate 
phylogenetic analysis was performed to assess the evolutionary origin in relation to the host of origin, the country of the phylogeographic genesis and the time for the most recent common ancestor (tMRCA) in order to determine the probable zoonotic origin.

The potential canine evolutionary origin for sample 143 appears evident in regards to the VP7, VP4, VP2 and NSP5/6 genes, all of which originating from the USA: the G3 genotype that has tMRCA in the year 1964, the P[3] tMRCA is in 1937, I3 have tMRCA in 1976 and the H6 genotype with tMRCA in year 1965. The C2 genotype has tMRCA of the year 1974 and origin in Australia. The NSP3 gene has originated from simian samples from Italy with T3 genotype and tMRCA in the year 1849. The NSP4 gene was identified as E3 genotype from Japan felines with tMRCA in the year 1974. Mutation rates ranged from $8,47^{\mathrm{E}-4}$ to $1.01^{\mathrm{E}-4}$ changes per site per year. The VP6 and NSP2 genes indicated human origin as denoted from I3 genotypes (tMRCA = 1976 - USA) and N2 (tMRCA = 1965 - USA), respectively (Figure 1-3).

Altogether 52127, 56010 and 55018 samples had a VP2 gene originating from China with chiroptera origin, and tMRCA in the year 1777. The analysis of the NSP1 gene yielded a A3 genotype with bovine evolution origin in the year of 1860 from the USA. NSP3, NSP4 and NSP5/6 genes were identified to be of feline origin, all which originating from Japan. In addition, T3 genotype had tMRCA in the year 1985, and those with E3 genotype presented tMRCA in 1855; 55127 and 56010 samples showed a H6 reassortant genotype with tMRCA in year 1939. Furthermore, 55018 sample was shown to be a with $\mathrm{H} 3$ genotype with tMRCA in the year 1936. Mutation rates ranged from $2.28^{\mathrm{E}-3}$ to $7.2^{\mathrm{E}-4}$ changes per site per year. VP7, VP4, VP6, VP1, VP3, NSP2 and NPS3 genes presented human origin with the genotypes G12
$($ tMRCA $=$ 1991-BRA), $\mathrm{P}$ [9] $($ TMRCA = 1997-BRA), I3 (tMRCA=1996-BRA), R3 (tMRCA $=1980-B R A)$, M3 (tMRCA = 1999BRA) and N3 (tMRCA = 1998-BRA), respectively (Figure 1-3).

The phylodynamic approach used in the present study sought to understand the relations and evolutionary origins. In this context, for instance, it was observed that samples belonging to the G4P[6] genotypes of porcine origin have frequently been described elsewhere, particularly in the Amazon region of Brazil (Maestri et al., 2012; Mascarenhas et al., 2007). In our study, phylodynamic analyzes showed that G4P[6] samples retained their human origin for the VP6 and NSP1 genes. It should be pointed out that VP6 is a key gene for the internalization of viral RNA, whereas NSP1 gene acts as an interferon antagonist (Carter and Saunders, 2007). Of note, our COD424 sample, denoted a reassortment involving the NSP3 gene, having the T7 genotype shown a similarity with the BE2001 strain of human-porcine origin. A few reassortment events like this, involving of human origin, have been described previously (Zeller et al., 2012). In our study, the HSE005 sample was shown to belong to the E1 genotype, lineage I, unlike G12P[6] strains described previously by Rahman et al., (2007) which belonged to lineage II.

The G3P[3] genotype found in sample 143 is well described in canines and felines, as well as in cattle, horses, chiropters, non-human primates and rodents (De Grazia et al., 2007; Sachsenröder et al., 2014; Sasaki et al., 2016); however, this genotype is rarely found in humans. Several studies have shown that G3P[3] is found mainly in strains of both canine and feline origins and it may also be identified in strains of human and in these animals origin (Luchs and Timenetsky, 2013). The G3P[3] sample showed the earliest evolutionary origin related to the NSP3 gene 
for the year 1849. In addition, we found that one of its evolutionary origins included a passage through apes. The remainder of its genes indicated an intense flow of evolutionary origins including strains of canine, feline and human origins. These findings suggest an ancient circulation between humans and domestic animals. The possible origin among felines and canines has already been reported elsewhere. Our analyses provide additional evidence on this (Luchs et al., 2012).

Samples 52127, 55018 and 56010, which shared the G12P[9] genotype-specificity, appeared to be of diverse evolutionary origins including human, chiropter, bovines and felines. The evolutionary origin of the chiropter, as denoted from the VP2 gene is not usual for G12P[9]. Our study indicates that evolutionary origin occurred in 1777, presenting a distant evolutionary past with origin in chiropter, therefore, this zoonotic characteristic is not usually detected using similarity or phylogenetic analysis, only when phylodynamic analysis was used. Donato et al., (2014) have described a sample obtained from an Australian child showing a remote similarity, as based on the VP2 gene analysis, with of bat-origin prototype MSLH14. These authors suggest that this may represent a possible mutant with escape from human vaccine and emphasize the need for a more comprehensive study aiming to determine the evolutionary origin of the MSLH14 strain. This evolutionary origin of G12P[9] strains was evidenced in the present study, their remote zoonotic evolutionary origin may be a determining factor in their current epidemiology. The bovine origin was evidence through analysis of the NSP1 gene, as already described in Brazil by Gómez et al., (2014) with the prototype E15776 G12P[9]. This prototype was generated through a reassortment which evidenced a bovine evolutionary origin, similarly to our findings in the present study all of which deriving from a common ancestor with tMRCA in the year 1860. Gómez et al., emphasize the fact that G12P[9] bears distinct zoonotic characteristics.

Table.1 Genotypes of the analyzed samples by phylogeny approach

\begin{tabular}{|c|c|c|c|c|c|c|c|c|c|c|c|}
\hline Sample & VP7 & VP4 & VP6 & VP1 & VP2 & VP3 & NSP1 & NSP2 & NSP3 & NSP4 & NSP5/6 \\
\hline 143 & G3 & P[3] & I3 & Rx & C2 & Mx & Ax & N2 & T3 & E3 & H6 \\
\hline COD055 & G4 & P[6] & I1 & R1 & C1 & M1 & A1 & N1 & T1 & E1 & H1 \\
\hline COD220 & G4 & P[x] & I1 & R1 & Cx & Mx & Ax & N1 & T1 & E1 & H1 \\
\hline COD379 & G4 & P[6] & I1 & R1 & Cx & M1 & A1 & N1 & T1 & E1 & H1 \\
\hline COD424 & G4 & P[6] & I1 & R1 & C1 & M1 & A1 & N1 & T7 & E1 & H1 \\
\hline HSE005 & G4 & P[6] & I2 & R2 & C2 & M2 & A2 & N2 & T2 & E1 & H2 \\
\hline $\mathbf{5 2 1 2 7}$ & G12 & P[9] & I3 & R3 & C3 & M3 & A3 & N3 & T3 & E3 & H6 \\
\hline $\mathbf{5 6 0 1 0}$ & G12 & P[9] & I3 & R3 & C3 & M3 & A3 & N3 & T3 & E3 & H6 \\
\hline $\mathbf{5 5 0 1 8}$ & G12 & P[9] & I3 & R3 & C3 & M3 & A3 & N3 & T3 & E3 & H3 \\
\hline
\end{tabular}

The colors represent the evolutionary origins: dark blue relates to feline origin; light blue to bat origin; red to canine origin; green to bovine origin; dark gray to porcine origin; and light gray to human origin. Genotypes with reassortment are indicated in yellow letters. 
Fig.1 Phylodynamic analysis of the VP7, VP4 and VP2 genes
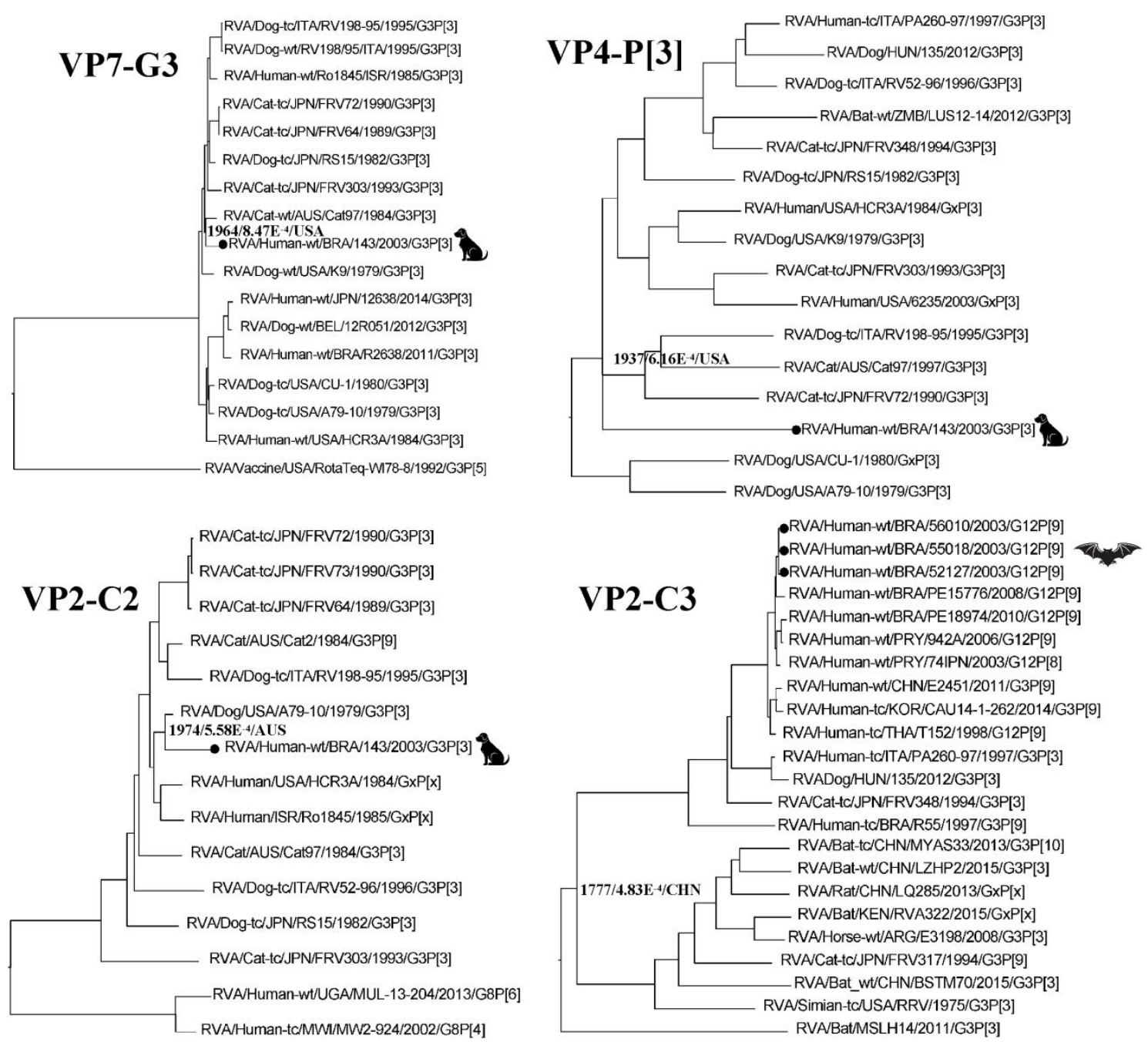

This analysis was performed for each of the genotypes of the zoonotic samples identified, showing the tMRCA, the mutation rate, the country of origin and the host of origin. Samples of the study are indicated by circles 
Fig.2 Phylodynamic analysis of the NSP1, NSP3, NSP4 and NSP5 genes
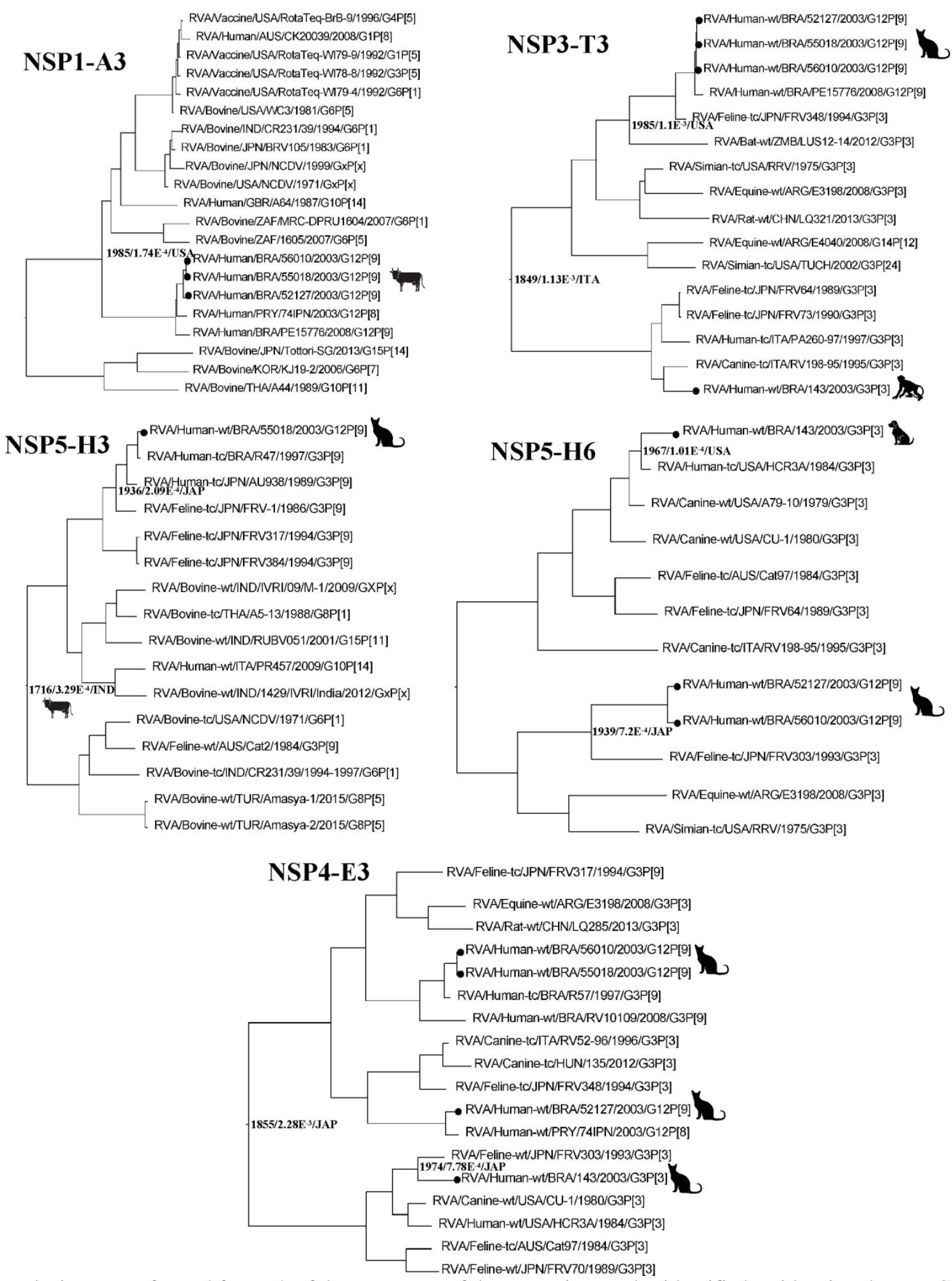

This analysis was performed for each of the genotypes of the zoonotic samples identified, evidencing the tMRCA, the mutation rate, the origin country and the host of origin. Samples of the study are indicated by circles 
Fig.3 Map of phylodynamic of zoonotic samples 143, 52127, 56010 and 55018

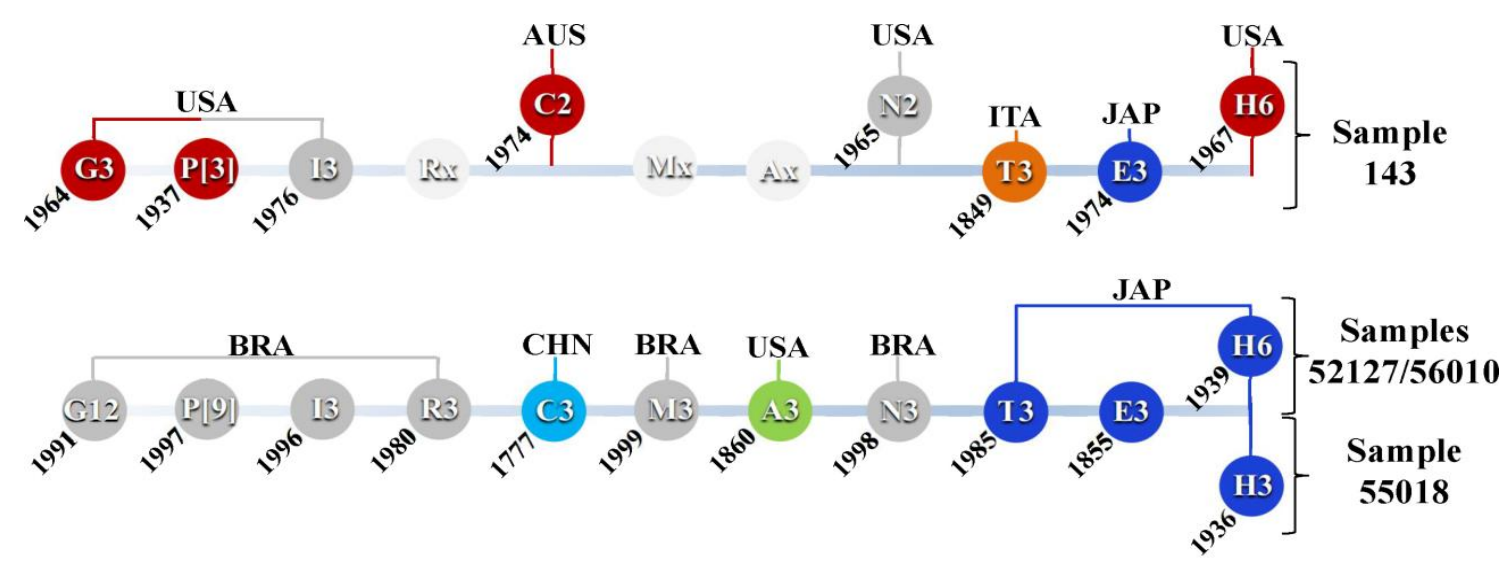

Legend:

Not genotyped Human Canine Bovine Feline Bat Simian

The colors represent the evolutionary origin according to the legend. The highlighted circles of the straight are the genotypes with reassortment, the numbers below the circles are the tMRCA of zoonotic evolutionary origin, above the circles are the countries of origin

The NSP3 gene of G12P[9] strains presented the T3 genotype with evolutionary origin in felines from Japan and tMRCA in the year 1985. Therefore, our samples are evolutionarily close to the feline FRV348 G3P[3] prototype isolated in Japan. For the NSP4 gene, the E3 genotype was also observed related to feline origin in Japan and with tMRCA in the year 1855. In regard to this gene samples were related to feline, canine, rodent and equine prototypes of the G3P[3] genotype, as well as with the human prototype 74IPN G12P[8]. The NSP5 gene has also denoted a feline origin from Japan; however, these samples have two distinct genotypes: samples 52127 and $56010 \mathrm{H} 6$ genotype with tMRCA in the year 1939, and sample 55018, H3 genotype, with tMRCA in the year 1936. The analysis of the NSP3, NSP4 and NSP5 genes indicated a feline G3P[3] origin. The feline origin of these genes had already been reported by Okitsu et al., (2018) in G3P[3] samples from diarrhoeic children. This evolutionary correlation with possible feline origin for G12P[9] had already been detected but not described and studied in detail (Gómez et al., 2014). Of note, Rhaman et al., (2007) have suggested that the evolutionary history of the G12P[9] genotype should be fully understood with further phylodynamic analyzes. In this regard, our study has provided the evidence towards the feline evolutionary origin of this strain.

The present study described the occurrence of four pattern of RVA zoonotic infection involving genes of both human and animal origins by analyzing samples from children with diarrhea, evidenced a well-established evolutionary exchange between interspecific hosts. Although previous studies in the Amazon have reported the occurrence of zoonotic evolutionary origin involving circulating rotavirus A strains in diarrhoeic children, our study provided additional information in this regard by using the phylodynamic approach. This accounted for a best understanding of the past evolutionary origin, of distinct strains, mainly those bearing $\mathrm{G} 3 \mathrm{P}[3]$ and G12P[9] genotype-specificities.

\section{Funding}

This research was supported by the Coordination of Improvement of Higher-Level Personnel (CAPES) n. 2303800717 / 2013-52, 
National Council for Scientific and Technological Development (CNPq), Evandro Chagas Institute, Ministry of Health, Ananindeua, Brazil and Amazon Foundation for Studies and Research (FAPESPA) for funding (ICAAF166 / 2014). SOUTO LCS is the beneficiary of the Capes and FAPESPA concession; SOUSA LC, and MASCARENHAS JDP are beneficiaries of grants from $\mathrm{CNPq}$.

\section{Acknowledgements}

We would like to thank to the Postgraduate Virology Program and the Evandro Chagas Institute for technical support to conclude this study, my counselor Joana Mascarenhas, my students Lizandra Souto and Layse Souza and all Rotavirus lab technicians for the immense contribution in the development of this article. To Dr. Márcio Nunes Head of the Laboratory of Genomics and other CIT-IEC technicians for the NGS sequencing.

\section{References}

Bezerra, D., Guerra, S., Serra, A., Fecury, P., Bandeira, R., Penha, E., Lobo, P., Sousa, E., Linhares, A., Soares, L., Mascarenhas, J. 2016. Analysis of a genotype G3P[9] rotavirus a strain that shows evidence of multiple reassortment events between animal and human rotaviruses. Journal of Medical Virology 89, 974-981. doi:10.1002/jmv.24733.

Boom, R., Sol, C. J., Salimans, M. M., Jansen, C. L., Wertheim-Van Dillen, P. M., Van Der Noordaa, J., 1990. Rapid and simple method for purification of nucleic acids. Journal of Clinical Microbiology 28, 495-503.

Bányai, K., Kemenesi, G., Budinski, I., Földes, F., Zana, B., Marton, S., Varga-Kugler, R., Oldal, M., Kurucz, K., Jakab, F., 2017. Candidate new rotavirus species in Schreiber's bats, Serbia. Infection, Genetics and Evolution 48, 19-26. doi:10.1016/j.meegid.2016.12.002.

Carter, J., Saunders, V. A., 2007. Virology: principles and applications. John Wiley \& Sons.

da Silva, M., Tort, L., Gómez, M., Assis, R., de Mendonça, M., Volotão, E., Leite, J., 2011. Phylogenetic analysis of VP1, VP2, and VP3 gene segments of genotype G5 group A rotavirus strains circulating in Brazil between 1986 and 2005. Virus Research 160, 381-388. doi:10.1016/j.virusres.2011.07.015.

De Grazia, S., Martella, V., Giammanco, G., Gòmara, M., Ramirez, S., Cascio, A., Colomba, C., Arista, S., 2007. CanineOrigin G3P[3] Rotavirus Strain in Child with Acute Gastroenteritis. Emerging Infectious Diseases 13, 1091-1093. doi:10.3201/eid1307.070239.

Dhama, K., Chauhan, R., Mahendran, M., Malik, S., 2008. Rotavirus diarrhea in bovines and other domestic animals. Veterinary Research Communications 33, 1-23. doi:10.1007/s11259-0089070-x.

Donato, C., Manuelpillai, N., Cowley, D., Roczo-Farkas, S., Buttery, J., Crawford, N., Kirkwood, C., 2014. Genetic characterization of a novel G3P[14] rotavirus strain causing gastroenteritis in 12year old Australian child. Infection, Genetics and Evolution 25, 97-109. doi:10.1016/j. meegid.2014.04.009.

Dóró, R., Farkas, S., Martella, V., Bányai, K., 2015. Zoonotic transmission of rotavirus: surveillance and control. Expert Review of Anti-infective Therapy 13, 1337-1350. doi:10.1586/14787210.2015.1089171.

Estes, M. K. Greenberg, H. B. Rotaviruses. In: Knipe, D. M., Howley, P. M., 
2013.Fields Virology. 6 ed. Lippincott, Williams and Wilkins; Philadelphia.1347-1401.

Gabbay, Y., Leite, J., Oliveira, D., Nakamura, L., Nunes, M., Mascarenhas, J., Heinemann, M., Linhares, A., 2007. Molecular epidemiology of astrovirus type 1 in Belém, Brazil, as an agent of infantile gastroenteritis, over a period of 18 years (1982-2000): Identification of two possible new lineages. Virus Research 129, 166-174. doi:10.1016/j. virusres.2007.07.006.

Gentsch, J., Laird, A., Bielfelt, B., Griffin, D., Bányai, K., Ramachandran, M., Jain, V., Cunliffe, N., Nakagomi, O., Kirkwood, C., Fischer, T., Parashar, U., Bresee, J., Jiang, B., Glass, R., 2005. Serotype Diversity and Reassortment between Human and Animal Rotavirus Strains: Implications for Rotavirus Vaccine Programs. The Journal of Infectious Diseases 192, S146-S159. doi:10.1086/431499.

Guerra, S., Soares, L., Lobo, P., Penha Júnior, E., Sousa Júnior, E., Bezerra, D., Vaz, L., Linhares, A., Mascarenhas, J., 2016. Detection of a novel equine-like G3 rotavirus associated with acute gastroenteritis in Brazil. Journal of General Virology 97, 3131-3138. doi:10.1099/jgv.0.000626.

Gómez, M., Resque, H., Volotão, E., Rose, T., Figueira Marques da Silva, M., Heylen, E., Zeller, M., Matthijnssens, J., Leite, J., 2014. Distinct evolutionary origins of G12P[8] and G12P[9] group A rotavirus strains circulating in Brazil. Infection, Genetics and Evolution 28, 385-388. doi:10.1016/j.meegid.2014.04.007.

Gómez, M., Resque, H., Volotão, E., Rose, T., Figueira Marques da Silva, M., Heylen, E., Zeller, M., Matthijnssens, J., Leite, J., 2014. Distinct evolutionary origins of G12P[8] and
G12P[9] group A rotavirus strains circulating in Brazil. Infection, Genetics and Evolution 28, 385-388. doi:10.1016/j.meegid.2014.04.007.

Huson, D., Bryant, D., 2005. Application of Phylogenetic Networks in Evolutionary Studies. Molecular Biology and Evolution 23, 254-267. doi:10.1093/molbev/msj030.

Katoh, K., Standley, D., 2013. MAFFT Multiple Sequence Alignment Software Version 7: Improvements in Performance and Usability. Molecular Biology and Evolution 30, 772-780. doi:10.1093/molbev/mst010.

Kearse, M., Moir, R., Wilson, A., StonesHavas, S., Cheung, M., Sturrock, S., Buxton, S., Cooper, A., Markowitz, S., Duran, C., Thierer, T., Ashton, B., Meintjes, P., Drummond, A., 2012. Geneious Basic: An integrated and extendable desktop software platform for the organization and analysis of sequence data. Bioinformatics 28, 1647-1649. doi:10.1093/ bioinformatics/bts199.

Khamrin, P., Maneekarn, N., Peerakome, S., Yagyu, F., Okitsu, S., Ushijima, H., 2006. Molecular characterization of a rare G3P[3] human rotavirus reassortant strain reveals evidence for multiple human-animal interspecies transmissions. Journal of Medical Virology $\quad 78, \quad 986-994$. doi:10.1002/jmv.20651.

Kindler, E., Trojnar, E., Heckel, G., Otto, P., Johne, R., 2013. Analysis of rotavirus species diversity and evolution including the newly determined fulllength genome sequences of rotavirus $F$ and G. Infection, Genetics and Evolution 14, 58-67. doi:10.1016/j.meegid.2012.11.015.

Larsson, A., 2014. AliView: a fast and lightweight alignment viewer and editor for large datasets. 
Bioinformatics $\quad 30, \quad 3276-3278$. doi:10.1093/bioinformatics/btu531.

Linhares, A. D. C., Gabbay, Y.B., Mascarenhas, J. D., De Freitas, R. B., Oliveira, C. S., Bellesi, N., Monteiro, T. A. F., Lins-Lainson, Z., Ramos, F. L. P., Valente, S. A., 1996. Immunogenicity, safety and efficacy of tetravalent rhesus-human, reassortant rotavirus vaccine in Belem, Brazil. Bull World Health Organization 74, 491.

Lole, K. S., Bollinger, R. C., Paranjape, R. S., Gadkari, D., Kulkarni, S. S., Novak, N. G., Ingersoll, R., Sheppard, H. W., Ray, S. C., 1999. Full-length human immunodeficiency virus type 1 genomes from subtype C-infected seroconverters in India, with evidence of intersubtype recombination. Journal of Virology 73, 152-60.

Luchs, A., Cilli, A., Morillo, S., Carmona, R., Timenetsky, M., 2012. Rare G3P[3] rotavirus strain detected in Brazil: Possible human-canine interspecies transmission. Journal of Clinical Virology $\quad 54, \quad 89-92$. doi:10.1016/j.jcv.2012.01.025.

Luchs, A., Timenetsky, M., 2013. Amino Acid sequence analysis of the two major outer Capsid Proteins (VP7 and VP4) from human-derived canine G3P[3] Rotavirus Strain Detected in Brazil. Journal of Health \& Biological Sciences 1, 145. doi:10.12662/23173076jhbs.v1i4.40.p145.2013.

Maestri, R., Kaiano, J., Neri, D., Soares, L., Guerra, S., Oliveira, D., Farias, Y., Gabbay, Y., Leite, J., Linhares, A., Mascarenhas, J., 2012. Phylogenetic analysis of probable Non-human genes of group A rotaviruses isolated from children with acute gastroenteritis in Belém, Brazil. Journal of Medical Virology 84, 1993-2002. doi:10.1002/jmv.23364.
Martella, V., Bányai, K., Matthijnssens, J., Buonavoglia, C., Ciarlet, M., 2010. Zoonotic aspects of rotaviruses. Veterinary Microbiology 140, 246255.

doi:10.1016/j.vetmic.2009.08.028.

Martin, D., Murrell, B., Golden, M., Khoosal, A., Muhire, B., 2015. RDP4: Detection and analysis of recombination patterns in virus genomes. Virus Evolution 1. doi:10.1093/ve/vev003.

Marton, S., Dóró, R., Fehér, E., Forró, B., Ihász, K., Varga-Kugler, R., Farkas, S., Bányai, K., 2017. Whole genome sequencing of a rare rotavirus from archived stool sample demonstrates independent zoonotic origin of human G8P[14] strains in Hungary. Virus Research 227, 96-103. doi:10.1016/j. virusres.2016.09.012.

Mascarenhas, J., Leite, J., Lima, J., Heinemann, M., Oliveira, D., Araujo, I., Soares, L., Gusmao, R., Gabbay, Y., Linhares, A., 2007. Detection of a neonatal human rotavirus strain with VP4 and NSP4 genes of porcine origin. Journal of Medical Microbiology 56, 524-532. doi:10.1099/jmm.0.46635-0.

Mascarenhas, J., Linhares, A., Gabbay, Y., Leite, J., 2002. Detection and Characterization of Rotavirus $\mathrm{G}$ and $\mathrm{P}$ Types from Children Participating in a Rotavirus Vaccine Trial in Belém, Brazil. Memórias do Instituto Oswaldo Cruz 97, 113-117. doi:10.1590/s007402762002000100020.

Matthijnssens, J., Ciarlet, M., Heiman, E., Arijs, I., Delbeke, T., McDonald, S., Palombo, E., Iturriza-Gomara, M., Maes, P., Patton, J., Rahman, M., Van Ranst, M., 2008. Full Genome-Based Classification of Rotaviruses Reveals a Common Origin between Human WaLike and Porcine Rotavirus Strains and Human DS-1-Like and Bovine 
Rotavirus Strains. Journal of Virology 82, 3204-3219. doi:10.1128/jvi.0225707.

Matthijnssens, J., Ciarlet, M., McDonald, S., Attoui, H., Bányai, K., Brister, J., Buesa, J., Esona, M., Estes, M., Gentsch, J., Iturriza-Gómara, M., Johne, R., Kirkwood, C., Martella, V., Mertens, P., Nakagomi, O., Parreño, V., Rahman, M., Ruggeri, F., Saif, L., Santos, N., Steyer, A., Taniguchi, K., Patton, J., Desselberger, U., Van Ranst, M., 2011. Uniformity of rotavirus strain nomenclature proposed by the Rotavirus Classification Working Group (RCWG). Archives of Virology 156, 1397-1413. doi:10.1007/s00705-011-1006-z.

Matthijnssens, J., Van Ranst, M., 2012. Genotype constellation and evolution of group A rotaviruses infecting humans. Current Opinion in Virology 2 , 426-433. doi:10.1016/j.coviro.2012.04.007.

Midgley, S., Böttiger, B., Jensen, T., FriisMøller, A., Person, L., Nielsen, L., Barzinci, S., Fischer, T., 2014. Human group A rotavirus infections in children in Denmark; detection of reassortant G9 strains and zoonotic $\mathrm{P}[14]$ strains. Infection, Genetics and Evolution 27, 114-120. doi:10.1016/j.meegid.2014.07.008.

Mihalov-Kovács, E., Gellért, Á., Marton, S., Farkas, S., Fehér, E., Oldal, M., Jakab, F., Martella, V., Bányai, K., 2015. Candidate New Rotavirus Species in Sheltered Dogs, Hungary. Emerging Infectious Diseases 21, 660-663. doi:10.3201/eid2104.141370.

Nakagomi, T., Agbemabiese, C., Nakagomi, O., 2018. Full genotype constellations of six feline Rotavirus A strains isolated in Japan in the 1990s including a rare A15 NSP1 genotype. Archives of Virology 163, 2257-2260. doi:10.1007/s00705-018-3835-5.

Nguyen, L., Schmidt, H., von Haeseler, A., Minh, B., 2014. IQ-TREE: A Fast and Effective Stochastic Algorithm for Estimating Maximum-Likelihood Phylogenies. Molecular Biology and Evolution 32, 268-274. doi:10.1093/molbev/msu300.

Okitsu, S., Hikita, T., Thongprachum, A., Khamrin, P., Takanashi, S., Hayakawa, S., Maneekarn, N., Ushijima, H., 2018. Detection and molecular characterization of two rare G8P[14] and $\mathrm{G} 3 \mathrm{P}[3]$ rotavirus strains collected from children with acute gastroenteritis in Japan. Infection, Genetics and Evolution 62, 95-108. doi:10.1016/j.meegid.2018.04.011.

Rahman, M., Matthijnssens, J., Yang, X., Delbeke, T., Arijs, I., Taniguchi, K., Iturriza-Gomara, M., Iftekharuddin, N., Azim, T., Van Ranst, M., 2006. Evolutionary History and Global Spread of the Emerging G12 Human Rotaviruses. Journal of Virology 81, 2382-2390. doi:10.1128/jvi.01622-06.

Rambaut A, Suchard MA, Xie D, Drummond AJ (2014) Tracer v1.6, Available from: http://beast.bio.ed.ac.uk/Tracer. Accessed 30 December 2019.

Rambaut A. (2009) FigTree, Version 1.4.2 Computer Program Distributed by the Author. http://tree.bio.ed.ac.uk/software/figtree I. Accessed 30 December 2019.

Rambaut, A., Lam, T., Max Carvalho, L., Pybus, O., 2016. Exploring the temporal structure of heterochronous sequences using TempEst (formerly Path-O-Gen). Virus Evolution 2, vew007. doi:10.1093/ve/vew007.

Sachsenroder, J., Braun, A., Machnowska, P., Ng, T., Deng, X., Guenther, S., Bernstein, S., Ulrich, R., Delwart, E., Johne, R., 2014. Metagenomic identification of novel enteric viruses 
in urban wild rats and genome characterization of a group A rotavirus. Journal of General Virology 95, 27342747. doi:10.1099/vir.0.070029-0.

Santos, N., Lima, R. C. C., Nozawa, C. M., Linhares, R. E., Gouvea, V., 1999. Detection of Porcine Rotavirus Type G9 and of a Mixture of Types G1 and G5 Associated with Wa-Like VP4 Specificity: Evidence for Natural Human-Porcine Genetic Reassortment. Journal of Clinical Microbiology 37, 2734-2736.

Sasaki, M., Orba, Y., Sasaki, S., Gonzalez, G., Ishii, A., Hang'ombe, B., Mweene, A., Ito, K., Sawa, H., 2016. Multireassortant G3P[3] group A rotavirus in a horseshoe bat in Zambia. Journal of General Virology 97, 2488-2493. doi:10.1099/jgv.0.000591.

Soares, L., Mascarenhas, J., Gabbay, Y., Gusmão, R., Linhares, A., 2010. Molecular characterization of G1 human rotaviruses detected in children from Belém, Pará, Brazil. Revista PanAmazônica de Saúde 1. doi: $10.5123 / \mathrm{s} 2176-$ 62232010000100018.

Suchard, M., Lemey, P., Baele, G., Ayres, D., Drummond, A., Rambaut, A., 2018. Bayesian phylogenetic and phylodynamic data integration using BEAST 1.10. Virus Evolution 4. doi:10.1093/ve/vey016.

Tate, J., Burton, A., Boschi-Pinto, C., Parashar, U., 2016. Global, Regional, and National Estimates of Rotavirus Mortality in Children <5 Years of Age, 2000-2013. Clinical Infectious Diseases 62, S96-S105. doi:10.1093/cid/civ1013.

Trojnar, E., Reetz, J., Twardziok, S., Sachsenröder, J., Johne, R., Otto, P., 2013. Identification of an avian group A rotavirus containing a novel VP4 gene with a close relationship to those of mammalian rotaviruses. Journal of General Virology 94, 136-142. doi:10.1099/vir.0.047381-0.

Wolfe, N., Dunavan, C., Diamond, J., 2007. Origins of major human infectious diseases. Nature 447, 279-283. doi:10.1038/nature05775.

Zeller, M., Heylen, E., De Coster, S., Van Ranst, M., Matthijnssens, J., 2012. Full genome characterization of a porcinelike human G9P[6] rotavirus strain isolated from an infant in Belgium. Infection, Genetics and Evolution 12, 1492-1500. doi:10.1016/j.meegid. 2012.03.002.

\section{How to cite this article:}

Renato da Silva Bandeira, Lizandra Caroline dos Santos Souto, Layse Costa de Souza, Delana Andreza Melo Bezerra, Poliana da Silva Lemos, Alexandre C. Linhares and Joana D'Arc Pereira Mascarenhas. 2019. Detection of Zoonotic Rotavirus Transmission involving a Rare Genotype G3P[3] in the Amazon Region: A Phylodynamic Approach with Multiple Events of Reassortment and Evolutionary Origin. Int.J.Curr.Microbiol.App.Sci. 8(11): 2175-2188. doi: https://doi.org/10.20546/ijcmas.2019.811.252 